\title{
ORTHOGONAL POLYNOMIALS ON THE SPHERE WITH OCTAHEDRAL SYMMETRY
}

BY

\author{
CHARLES F. DUNKL ${ }^{1}$
}

\begin{abstract}
For any finite reflection group $G$ acting on $\mathbf{R}^{N}$ there is a family of $G$-invariant measures $\left(h^{2} d \omega\right.$, where $h$ is a certain product of linear functions whose zero-sets are the reflecting hyperplanes for $G$ ) on the unit sphere and an associated partial differential operator $\left(L_{h} f:=\Delta(f h)-f \Delta h ; \Delta\right.$ is the Laplacian). Analogously to spherical harmonics, there is an orthogonal (with respect to $h^{2} d \omega$ ) decomposition of homogeneous polynomials, that is, if $p$ is of degree $n$ then

$$
p(x)=\sum_{i=0}^{[n / 2]}|x|^{2 j} p_{n-2 j}(x),
$$

where $L_{h} p_{i}=0$ and $\operatorname{deg} p_{i}=i$ for each $i$, but with the restriction that $p$ and $p_{i}$ must all be $G$-invariant. The main topic is the hyperoctahedral group with

$$
h(x)=\left(x_{1} x_{2} \cdots x_{N}\right)^{\alpha}\left(\prod_{i<j}\left(x_{i}^{2}-x_{j}^{2}\right)\right)^{\beta} \text {. }
$$

The special case $N=2$ leads to Jacobi polynomials. A detailed study of the case $N=3$ is made; an important result is the construction of a third-order differential operator that maps polynomials associated to $h$ with indices $(\alpha, \beta)$ to those associated with $(\alpha+2, \beta+1)$.
\end{abstract}

Introduction. Regular figures have long been objects of fascination. We study here the interplay between them and the theory of orthogonal polynomials. This will also touch on finite groups generated by reflections and their polynomial invariants, as well as a generalization of spherical harmonics. The underlying situation throughout will consist of spaces of homogeneous polynomials on $\mathbf{R}^{N}$ that satisfy a second-order differential equation and whose restrictions to the unit sphere have an orthogonality relation with respect to a weight function, and of a finite reflection group for which all these objects are invariant.

The weight (measure) $h^{2}(x) d \omega(x)$ on the unit sphere $S$ in $\mathbf{R}^{3}$, where

$$
h(x)=\left(x_{1} x_{2} x_{3}\right)^{\alpha}\left(\left(x_{1}^{2}-x_{2}^{2}\right)\left(x_{1}^{2}-x_{3}^{2}\right)\left(x_{2}^{2}-x_{3}^{2}\right)\right)^{\beta},
$$

$\alpha, \beta=1,2, \ldots(d \omega$ is surface area), and the associated polynomials will be studied most intensely. Some of the highlights of the theory are as follows:

Define the differential operator

$$
L_{h} f:=\Delta(f h)-f \Delta h
$$

Received by the editors April 19, 1982 and, in revised form, December 15, 1982.

1980 Mathematics Subject Classification. Primary 33A45, 33A65; Secondary 20H15.

'This research was partially supported by NSF Grant MCS 81-02581.

(C) 1984 American Mathematical Society $0002-9947 / 84 \$ 1.00+\$ .25$ per page 
(for $f$ smooth on $\mathbf{R}^{3}$, and $\Delta$ is the Laplacian). Then any polynomial solution $p$ of $L_{h} p=0$ is invariant under the octahedral group and hence is a polynomial in $e_{1}, e_{2}, e_{3}$ where

$$
e_{1}:=\sum_{i} x_{i}^{2}, \quad e_{2}:=\sum_{i<j} x_{i}^{2} x_{j}^{2}, \quad e_{3}:=x_{1}^{2} x_{2}^{2} x_{3}^{2}
$$

Furthermore, if $p$ and $q$ are homogeneous polynomials of different degrees, $L_{h} p=0$ and $L_{h} q=0$, then $\int_{S} p q h^{2} d \omega=0$. The subset $\left\{\left(x_{1}, x_{2}, x_{3}\right) \in S: x_{1} \geqslant x_{2} \geqslant x_{3} \geqslant 0\right\}$ corresponds to the region $E:=\left\{\left(e_{2}, e_{3}\right): e_{2}, e_{3}, D \geqslant 0\right\}$ where $D=e_{2}^{2}-4 e_{2}^{3}-4 e_{3}$ $-27 e_{3}^{2}+18 e_{2} e_{3}$ (the restriction of the discriminant), and the measure $h^{2}(x) d \omega(x)$ corresponds to $\frac{3}{\pi} e_{3}^{\alpha-1 / 2} D^{\beta-1 / 2} d e_{2} d e_{3}$ on $E$.

In a sense we consider orthogonal polynomials on $E$, but there is a significant difference between this situation and the usual one. In the latter the $x$ and $y$ variables are each given degree 1 , whereas here $e_{2}$ and $e_{3}$ are of degrees 2 and 3 , respectively (in $\left\{x_{i}^{2}\right\}$ ), so that, for example, $e_{2}^{3}+e_{3}^{2}$ is homogeneous of degree 6 .

The statements about $L_{h}$ and $h^{2} d \omega$ actually can be applied to any finite reflection group on $\mathbf{R}^{N}$, and in $\S 2$ this general situation will be presented. The key observation is that $h$ is a product of linear functions such that the reflections in their zero sets (hyperplanes) generate a finite reflection group under which $h$ is (relatively) invariant. The relation of this to ordinary harmonic polynomials will also be considered.

In $\S 3$ the general theory will be applied to the function

$$
h(x)=\left(x_{1} x_{2} \cdots x_{N}\right)^{\alpha} \cdot\left(\prod_{i<j}\left(x_{i}^{2}-x_{j}^{2}\right)\right)^{\beta}
$$

on $\mathbf{R}^{N}(\alpha, \beta=1,2, \ldots)$, and solutions of $L_{h} p=0$ will be found in terms of $e_{i}$ $(1 \leqslant i \leqslant N)$, where $e_{i}$ is the elementary symmetric function of degree $i$ in $x_{1}^{2}, x_{2}^{2}, \ldots, x_{N}^{2}$.

Finally a detailed investigation of this function $h$ will be made in the cases $N=2$ and $N=3$. We present the $N=2$ version, which is essentially the theory of Jacobi polynomials, by way of motivation for further studies. For $N=3$ we describe a third-order differential operator which maps polynomials associated to the parameters $(\alpha, \beta)$ to those for $(\alpha+2, \beta+1)$ (analogously to the derivative of $P_{n}^{(\alpha, \beta)}$ being a multiple of $P_{n-1}^{(\alpha+1, \beta+1)}$, for Jacobi polynomials).

Notation and conventions: For an element $x=\left(x_{1}, x_{2}, \ldots, x_{N}\right) \in \mathbf{R}^{N}$ the norm $|x|:=\left(\sum_{i} x_{i}^{2}\right)^{1 / 2}$. The unit sphere $S$ in $\mathbf{R}^{N}$ is the set $\{x:|x|=1\}$. The rotationinvariant surface measure $d \omega$ on $S$ is the normalized one, that is, $\int_{S} d \omega=1$.

All the functions will be real valued, while parameters such as $\alpha, \beta$ will be integer valued (extensions to real values of $\alpha, \beta$ are fairly obvious).

For integers $m, n$ let $m \wedge n:=\min (m, n), m \vee n:=\max (m, n)$. For a finite set $\Omega,|\Omega|$ denotes the cardinality. The Laplacian on $\mathbf{R}^{N}$ is $\Delta:=\sum_{i=1}^{N}\left(\partial / \partial x_{i}\right)^{2}$. For differential operators $D_{1}, D_{2}$ the commutator is $\left[D_{1}, D_{2}\right]:=D_{1} D_{2}-D_{2} D_{1}$. 
2. Reflection groups and the Laplacian. There is a close connection between polynomials on $\mathbf{R}^{N}$ which are products of linear functions, the Laplacian, and finite reflection groups.

2.1. Definition. A reflection group $G$ is a finite subgroup of the orthogonal group on $\mathbf{R}^{N}$, some $N \geqslant 1$, that is generated by its reflections. A reflection is an involutory orthogonal transformation that has just one eigenvalue of -1 (with the rest being 1 ). Each reflection is associated to its hyperplane of fixed points, which in turn is associated to a linear function, its annihilator (determined up to a constant). For $n \geqslant 0$ let $P_{n}^{G}$ be the space of homogeneous polynomials on $\mathbf{R}^{N}$ of degree $n$ that are invariant under $G$ (that is, $\left.p(\sigma x)=p(x)\left(\sigma \in G, x \in \mathbf{R}^{N}\right)\right)$.

We list the known results (see Stanley [11, pp. 485-488]) about reflection groups which are relevant to our study. There is a set of homogeneous invariant polynomials $\theta_{1}, \theta_{2}, \ldots, \theta_{N}$ of degrees $d_{1}, d_{2}, \ldots, d_{N}$ such that the ring of $G$-invariant polynomials $\left(\sum_{n \geqslant 0} \oplus P_{n}^{G}\right)$ is exactly the ring of all polynomials in $\theta_{1}, \ldots, \theta_{N}$, and $|G|=\prod_{i=1}^{N} d_{i}$. Furthermore, the Jacobian $\partial\left(\theta_{1}, \ldots, \theta_{N}\right) / \partial\left(x_{1}, \ldots, x_{N}\right)$ is a scalar multiple of the product of the linear functions corresponding to the reflections in $G$ (thus there are $\sum_{i=1}^{N}\left(d_{i}-1\right)$ of these) (for a quick proof, see Steinberg [12]). A reflection group is said to be irreducible if it cannot be decomposed as a product of two reflection groups acting on nontrivial orthogonal subspaces of $\mathbf{R}^{N}$. The irreducible reflection groups are known (see Coxeter [2, Chapter 11]) and include the groups of type $A_{N}$ (symmetric), $B_{N}$ (hyperoctahedral), $D_{N}$, acting on $\mathbf{R}^{N}$, the dihedral groups on $\mathbf{R}^{2}$, and a finite set of exceptional groups: $E_{6}, E_{7}, E_{8}, F_{4}, H_{3}$ (icosahedral), $H_{4}$.

2.2. Definition. Suppose $h$ is a product of powers of linear functions on $\mathbf{R}^{N}$. We say $h$ satisfies condition $(*)$ for the (finite) reflection group $G$ if the reflections corresponding to the linear functions in $h$ generate $G$ and $h(\sigma x)= \pm h(x)(\sigma \in G)$ (this property is called relative invariance).

Suppose the reflections $w_{j}(1 \leqslant j \leqslant m)$ of the reflection group $G$ are in the hyperplanes $\left\{x: l_{j}(x)=0\right\}$, respectively (by convention, $G$ fixes the origin so each $l_{j}$ is homogeneous), and are partitioned into conjugacy classes $C_{i}(1 \leqslant i \leqslant M)$. Then the most general $h$ satisfying (*) for $G$ is $\prod_{i=1}^{M}\left(\prod_{j \in C_{1}} l_{j}\right)^{\alpha_{i}}$ with each $\alpha_{i} \geqslant 1$. Note that if $w$ is the reflection in $\{x: l(x)=0\}$, then $\sigma^{-1} w \sigma$ is the reflection in $\{x: l(\sigma x)=0\}$ $(\sigma \in G)$.

Keeping in mind that we are concerned with weight functions on the sphere which are invariant under a reflection group, we now define a differential operator associated to such weights. The remarkable fact is that the polynomials in its kernel must themselves be invariant.

2.3. Definition. For $h \in C^{\infty}\left(\mathbf{R}^{N}\right)$ the linear operator $L_{h}$ is defined by $L_{h} f:=\Delta(f h)-f \Delta h\left(f \in C^{\infty}\left(\mathbf{R}^{N}\right)\right)$.

2.4. THEOREM. Let $l$ be a nontrivial linear function on $\mathbf{R}^{N}$ and $w$ the reflection in $\{x$ : $l(x)=0\}$. Suppose $h=l^{\alpha} g$, where $\alpha$ is a positive integer and $g$ is a polynomial invariant under $w$ and not divisible by $l$. If $f$ is a nontrivial polynomial on $\mathbf{R}^{N}$ such that $L_{h} f=0$, then $f$ is invariant under $w$ and not divisible by $l$. 
Proof. Since $\Delta$ is invariant under orthogonal change of basis and translation we may assume $l(x)=x_{N}$, so by hypothesis $g(x)$ is even in $x_{N}$ and not divisible by $x_{N}$. Let $f$ be a polynomial solution of $L_{h} f=0$ and write

$$
f(x)=\sum_{m=0}^{M} p_{m}\left(x_{1}, \ldots, x_{N-1}\right) x_{N}^{m} .
$$

By symmetry the transformed function $\check{f}(x)=\Sigma_{m} p_{m}(-1)^{m} x_{N}^{m}$ also satisfies the equation, and so does the odd part $f_{-}=(f-\check{f}) / 2$. For the invariance we must show that $f_{-}=0$. Suppose not, and let $p_{2 k+1}$ be the nonzero term of least index in $f_{-}$. Consider the expansion

$$
\begin{aligned}
\frac{1}{g(x) x_{N}^{\alpha}} L_{h} f_{-}=\sum_{m}\left\{2(2 m+1)(m+\alpha) p_{2 m+1} x_{N}^{2 m-1}\right. & \\
+\left(\Delta p_{2 m+1}+2 \sum_{i=1}^{N-1} \frac{1}{g} \frac{\partial g}{\partial x_{i}} \frac{\partial}{\partial x_{i}} p_{2 m+1}\right) x_{N}^{2 m+1} & \\
& \left.\quad+2(2 m+1) \frac{1}{g} \frac{\partial g}{\partial x_{N}} p_{2 m+1} x_{N}^{2 m}\right\} .
\end{aligned}
$$

The nonzero term with the least exponent of $x_{N}$ in this sum is

$$
2(2 k+1)(k+\alpha) p_{2 k+1} x_{N}^{2 k-1}
$$

( $x_{N}$ does not divide $g$ so the denominators are no problem), implying that $L_{h} f_{-} \neq 0$, a contradiction.

Now let $p_{2 k}$ be the nonzero term of least index in $f$. The coefficient of $x_{N}^{2 k-2}$ in $\left(1 / g x_{N}^{\alpha}\right) L_{h} f$ is $2 k(2 k-1+2 \alpha) p_{2 k}$, which can only vanish if $k=0$.

2.5. Corollary. Suppose $h$ satisfies condition (*) for the reflection group $G$, and $f$ is a polynomial satisfying $L_{h} f=0$. Then $f$ is invariant under $G$.

Proof. Apply the theorem to each (maximal power of a) linear factor in $h$.

The following was found for $\mathbf{R}^{3}$ by Laporte [5]. Since this is not an easily accessible paper, we give a general proof.

2.6. Corollary. Let $P$ be a product of (possibly inhomogeneous) linear functions on $\mathbf{R}^{N}$. Then $\Delta P=0$ if and only if the reflections in the hyperplanes corresponding to the factors in $P$ are exactly the set of reflections in a finite reflection group, and there are no repeated factors.

Proof. By translation and rotation we may assume one of the factors is $x_{N}$. Write $P(x)=x_{N} f(x)$ and note that $L_{h} f=0$ for $h(x)=x_{N}$. By the theorem $f$ is invariant under the reflection in $\left\{x_{N}=0\right\}$ and is not divisible by $x_{N}$ (thus, no repeated factors). The invariance shows that the reflecting hyperplanes are mapped into each other by the action of any one of the reflections.

Let $P_{0}$ be the product of the homogeneous (degree 1) parts of the factors of $P$, that is, the part of highest degree of $P$. Since $\Delta$ is homogeneous, $\Delta P_{0}=0$. Assume the orthogonal complements of the hyperplanes in $P_{0}$ span $\mathbf{R}^{N}$, so the reflection group $G$ 
generated by the reflections in $P_{0}$ is finite because it acts on the finite set of orthogonal complements. Since $G$ is a linear group, only the identity can fix all these subspaces.

Now the reflections in $P$ are translations of the reflections of $G$. They must have a common fixed point, or else the group generated by the reflections in $P$ would contain translations which produce infinitely many mirrors.

The converse is a well-known trick. Note that $P$ changes sign under the action of a single reflection, and so does $\Delta P$, but $\Delta P$ is of lower degree than $P$ and so cannot be nontrivially divisible by each linear function corresponding to a reflection in the group; hence $\Delta P=0$.

The orthogonality structure associated to $L_{h}$ depends on Green's theorem.

2.7. Lemma. Let $h \in C^{\infty}\left(\mathbf{R}^{N}\right)$ and suppose $f, g$ are homogeneous polynomials of different degrees and $L_{h} f=0, L_{h} g=0$. Then $\int_{S} f g h^{2} d \omega=0$.

Proof. By Green's second identity $(B:=\{|x|<1\})$

$$
\begin{aligned}
\int_{B}\left(g L_{h} f-f L_{h} g\right) h d x & =\int_{B}\{h g \Delta(h f)-h f \Delta(h g)\} d x \\
& =c_{N} \int_{S}\left(g \frac{\partial}{\partial n} f-f \frac{\partial}{\partial n} g\right) h^{2} d \omega \\
& =c_{N}(\operatorname{deg} f-\operatorname{deg} g) \int_{S} g h^{2} d \omega,
\end{aligned}
$$

where $\partial / \partial n$ is the normal derivative on $S$ and $c_{N}$ is the surface measure of $S$ in $\mathbf{R}^{N}$. For homogeneous polynomials $\partial f / \partial n=(\operatorname{deg} f) f$ on $S$.

Any homogeneous polynomial $p$ on $\mathbf{R}^{N}$ has the unique expansion

$$
p(x)=\sum_{j}|x|^{2 j} p_{n-2 j}(x),
$$

where $n=\operatorname{deg} p$ and $p_{n-2 j}$ is harmonic and homogeneous of degree $n-2 j$ (thus any polynomial restricted to $S$ is the boundary value of a harmonic polynomial). By Theorem 2.4 an analogous situation for the operator $L_{h}$ can only exist for invariant polynomials.

2.8. Definition. Let $G$ be a finite reflection group and let $h$ satisfy condition $(*)$ for $G$. Then $H_{n}^{h}:=\left\{p \in P_{n}^{G}: L_{h} p=0\right\}$ (the $G$-invariant polynomials of degree $n$ annihilated by $\left.L_{h}\right)(n=0,1,2, \ldots)$.

2.9. THEOREM. If h satisfies $(*)$ for $G$, then

$$
P_{n}^{G}=\sum_{j} \oplus|x|^{2 j} H_{n-2 j}^{h} \quad(n \geqslant 0) .
$$

Proof. From Lemma 2.7 we have $H_{n}^{h} \cap|x|^{2 j} H_{n-2 j}^{h}=\{0\}$ for $j>0$. This shows that $P_{n}^{G}$ contains the indicated spaces as direct summands. The modified operator

$$
\tilde{L}_{h}:=\frac{1}{h} L_{h}=\Delta+\frac{2}{h} \sum_{i=1}^{N} \frac{\partial h}{\partial x_{i}} \frac{\partial}{\partial x_{i}}
$$


maps $P_{n}^{G}$ into $P_{n-2}^{G}$. To see this, assume, as in Theorem 2.4, that $h(x)=x_{n} g(x)$ with $g$ even in $x_{N}$ and not divisible by $x_{N}$. If $f \in P_{n}^{G}$ then $f$ is even in $x_{N}$, and by the calculation in the proof of $2.4, \tilde{L}_{h} f$ is an even polynomial in $x_{N}$ with possibly rational coefficients, where the denominator is $g$. This argument is applied to every linear factor of $h$, and so $\tilde{L}_{h} f$ is a $G$-invariant polynomial. Clearly the degree of homogeneity of $\tilde{L}_{h} f$ is $n-2$.

Hence $\operatorname{dim} P_{n-2}^{G}+\operatorname{dim} H_{n}^{h} \geqslant \operatorname{dim} P_{n}^{G}$. Assume the theorem is true for $n \leqslant k-1$ (it is automatic for $k=2$ ). Then $H_{k}^{h} \oplus|x|^{2} P_{k-2}^{G}=\Sigma_{j} \oplus|x|^{2 j} H_{n-2 j}^{h}$ has dimension at least as large as $P_{k}^{G}$, hence is equal to $P_{k}^{G}$. By induction, the proof is finished.

Just as Prasad [9] (among others) gave an explicit expression for $p_{n-2 j}$ in the series $p(x)=\Sigma_{j}|x|^{2 j} p_{n-2 j}(x)$ with $p \in P_{n}$ and $\Delta p_{n-2 j}=0$, we can determine $p_{n-2 j}$ with $L_{h} p_{n-2 j}=0$, when $p$ is $G$-invariant.

2.10. Definition. For $n=0,1,2, \ldots$ define the operator

$$
T_{h, n} p(x):=\sum_{i=0}^{[n / 2]} \frac{|x|^{2 i}}{4^{i} i !(-N / 2-\operatorname{deg} h-n+2)_{i}} \tilde{L}_{h}^{i} p(x) \quad\left(p \in P_{n}^{G}\right)
$$

(recall $\left.\tilde{L}_{h}=(1 / h) L_{h}\right)$.

2.11. THEOREM. The operator $T_{h, n}$ is the orthogonal projection $P_{n}^{G} \rightarrow H_{n}^{h}$, and further, any $p \in P_{n}^{G}$ is expanded as

$$
p(x)=\sum_{j=0}^{[n / 2]}|x|^{2 j} p_{n-2 j}(x)
$$

with $p_{n-2 j} \in H_{n-2 j}^{h}$, where

$$
p_{n-2 j}:=\left(4^{j} j !(N / 2+\operatorname{deg} h+n-2 j)_{j}\right)^{-1} T_{h, n-2 j}\left(\tilde{L}_{h}^{j} p\right) .
$$

Proof. From the identity

$$
\Delta\left(|x|^{2 m} f\right)=4 m(N / 2+m-1+\operatorname{deg} f)|x|^{2 m-2} f+|x|^{2 m} \Delta f,
$$

for $f$ homogeneous, follows

$$
L_{h}\left(|x|^{2 m} p\right)=4 m(N / 2+m-1+\operatorname{deg} h+\operatorname{deg} p)|x|^{2 m-2} h p+|x|^{2 m} L_{h}(p) .
$$

Let $p \in P_{n}^{G}$. Then by Theorem 2.9,

$$
p=\sum_{j}|x|^{2 j} p_{n-2 j} \quad \text { with } p_{n-2 j} \in H_{n-2 j}^{h} .
$$

By repeated use of the formula for $L_{h}$ we obtain

$$
\tilde{L}_{h}^{m} p=\sum_{i=m}^{[n / 2]} 4^{m}(-i)_{m}(i-N / 2-\operatorname{deg} h-n+1)_{m}|x|^{2 i-2 m} p_{n-2 i} .
$$

It can now be directly verified that $T_{h, n} p=p_{n}$ (this uses the Chu-Vandermonde sum). (The form of $T_{h, n}$ was obtained after some calculations and judicious guessing.) Finally, note that $p_{n-2 m}$ is a multiple of the projection of $\tilde{L}_{h}^{m} p$ onto $H_{n-2 m}^{h}$, namely $T_{h, n-2 m} \tilde{L}_{h}^{m} p$.

The author thanks the referee for suggesting the use of powers of $\tilde{L}_{h}$ to calculate the $p_{n-2 j}$ 's. 
Koornwinder [4] used an operator essentially the same as $L_{h}$ in describing two-variable analogues of Jacobi polynomials. The refection groups he used either acted on the plane $\left(\mathbf{R}^{2}\right)$ or acted reducibly on $\mathbf{R}^{3}$.

3. The groups of type $\mathbf{B}_{\mathbf{N}}$ and $\mathbf{D}_{\mathrm{N}}$. Here we will be concerned with the function

$$
h(x)=\left(x_{1} x_{2} \cdots x_{N}\right)^{\alpha}\left(\prod_{i<j}\left(x_{i}^{2}-x_{j}^{2}\right)\right)^{\beta} \quad(\alpha, \beta \geqslant 1) .
$$

This satisfies condition (*) for $O_{N}$, the (full) hyperoctahedral group on $\mathbf{R}^{N}$, which is the symmetry group of the cube $\{( \pm 1, \pm 1, \ldots, \pm 1)\}$ and is generated by the reflections in $x_{i}=0, x_{i}+x_{j}=0, x_{i}-x_{j}=0 \quad(i, j=1, \ldots, N$ and $i \neq j)$. The invariants for $O_{N}$ are certainly even in each $x_{i}$, so for convenience we introduce variables $y_{i}:=x_{i}^{2}(1 \leqslant i \leqslant N)$ and express $\tilde{L}_{h}=(1 / h) L_{h}$ in terms of these.

3.1. PROPOSITION.

$$
\begin{aligned}
& \tilde{L}_{h}=\Delta+2 \alpha \sum_{i=1}^{N} \frac{1}{x_{i}} \frac{\partial}{\partial x_{i}}+4 \beta \sum_{i<j}\left(\frac{1}{x_{i}^{2}-x_{j}^{2}}\right)\left(x_{i} \frac{\partial}{\partial x_{i}}-x_{j} \frac{\partial}{\partial x_{j}}\right) \\
& \tilde{L}_{h}=4\left(\sum_{i=1}^{N} y_{i}\left(\frac{\partial}{\partial y_{i}}\right)^{2}+\left(\alpha+\frac{1}{2}\right) \sum_{i=1}^{N} \frac{\partial}{\partial y_{i}}+2 \beta \sum_{i<j}\left(\frac{1}{y_{i}-y_{j}}\right)\left(y_{i} \frac{\partial}{\partial y_{i}}-y_{j} \frac{\partial}{\partial y_{j}}\right)\right)
\end{aligned}
$$

in the $x$ and $y$ variables, respectively.

Proof. This is a direct computation using

and product formulas.

$$
\tilde{L}_{h}=\Delta+\frac{2}{h} \sum_{i=1}^{N}\left(\frac{\partial h}{\partial x_{i}}\right) \frac{\partial}{\partial x_{i}}
$$

3.2. Definition.

$$
\begin{gathered}
L:=\sum_{i=1}^{N} y_{i}\left(\frac{\partial}{\partial y_{i}}\right)^{2}, \quad \delta_{1}:=\sum_{i=1}^{N} \frac{\partial}{\partial y_{i}}, \\
\delta_{2}:=\sum_{1 \leqslant i<j \leqslant N}\left(\frac{1}{y_{i}-y_{j}}\right)\left(y_{i} \frac{\partial}{\partial y_{i}}-y_{j} \frac{\partial}{\partial y_{j}}\right) .
\end{gathered}
$$

Each of these differential operators lowers the degree of homogeneity (in $y$ ) by one. To indicate dependence on $\alpha, \beta$ we also define

$$
L_{\alpha \beta}:=L+\left(\alpha+\frac{1}{2}\right) \delta_{1}+2 \beta \delta_{2}=\frac{1}{4} \tilde{L}_{h} .
$$

It is known that the invariants of $O_{N}$ are the polynomials in the elementary symmetric functions in $\left\{x_{i}^{2}: 1 \leqslant i \leqslant N\right\}$, which we will denote by $e_{i}, 0 \leqslant i \leqslant N$. They are defined by the generating function

$$
\sum_{i=0}^{N} e_{i} t^{i}=\prod_{i=1}^{N}\left(1+y_{i} t\right)
$$

Further, for dealing with polynomials in the $e_{i}$, we will use the short forms $\partial_{j}:=\partial / \partial e_{j}$. 
3.3. Proposition. For $1 \leqslant j \leqslant N$,

$$
\delta_{1} e_{j}=(N-j+1) e_{j-1} \quad \text { and } \quad \delta_{2} e_{j}=\left(\begin{array}{c}
N-j+1 \\
2
\end{array}\right) e_{j-1}
$$

equivalently,

$$
\delta_{1}=\sum_{j=1}^{N}(N-j+1) e_{j-1} \partial_{j}, \quad \delta_{2}=\sum_{j=1}^{N-1}\left(\begin{array}{c}
N-j+1 \\
2
\end{array}\right) e_{j-1} \partial_{j} .
$$

Proof. We use a rather heavy-handed generating function approach since it gives neat proofs for these facts as well as for the next proposition. Let

$$
F:=\prod_{i=1}^{N}\left(t+y_{i}\right)=\sum_{i=0}^{N} e_{i} t^{N-i}
$$

Then

$$
\begin{aligned}
\delta_{1} F & =\sum_{i=0}^{N}\left(\delta_{1} e_{i}\right) t^{N-i}=\sum_{i=1}^{N} \prod_{j \neq i}\left(t+y_{i}\right)=\frac{\partial}{\partial t} F \\
& =\sum_{i=0}^{N-1} e_{i}(N-i) t^{N-i-1}=\sum_{i=1}^{N}(N-i+1) e_{i-1} t^{N-i} .
\end{aligned}
$$

Also

$$
\begin{aligned}
\delta_{2} F & =\sum_{i=0}^{N}\left(\delta_{2} e_{i}\right) t^{N-i} \\
& =\sum_{1 \leqslant i<j \leqslant N}\left(\frac{1}{y_{i}-y_{j}}\right)\left(y_{i} \frac{\partial}{\partial y_{i}}-y_{j} \frac{\partial}{\partial y_{j}}\right)\left(t+y_{i}\right)\left(t+y_{j}\right) \prod_{k \neq i, j}\left(t+y_{k}\right) \\
& =t \sum_{i<j} \prod_{k \neq i, j}\left(t+y_{k}\right)=\frac{t}{2} \sum_{i=1}^{N} \sum_{j \neq i} \prod_{k \neq i, j}\left(t+y_{k}\right) \\
& =\frac{t}{2}\left(\frac{\partial}{\partial t}\right)^{2} F=\sum_{i=1}^{N} \frac{(N-i+1)(N-i)}{2} e_{i-1} t^{N-i} \cdot \square
\end{aligned}
$$

3.4. Proposition. For polynomials in $\left\{e_{j}\right\}$,

$$
L=\sum_{i, j=1}^{N}\left(\sum_{k=i \vee j}^{N \wedge(i+j-1)}(2 k-i-j+1) e_{k} e_{i+j-k-1}\right) \partial_{i} \partial_{j} .
$$

Proof. First observe that

$$
L f\left(e_{1}, \ldots, e_{N}\right)=\sum_{i, j=1}^{N}\left(\sum_{k=1}^{N} y_{k} \frac{\partial e_{i}}{\partial y_{k}} \frac{\partial e_{j}}{\partial y_{k}}\right) \partial_{i} \partial_{j} f
$$

(since $\left(\partial / \partial y_{k}\right)^{2} e_{i}=0$ for all $\left.i, k\right)$. The $k$-sum is the coefficient of $s^{i} t^{j}$ in the sum

$$
\sum_{k=1}^{N} y_{k} s t \prod_{m \neq k}\left(1+s y_{m}\right)\left(1+t y_{m}\right)
$$


which equals

$$
\begin{aligned}
s t & \sum_{k=1}^{N} \frac{y_{k}}{\left(1+s y_{k}\right)\left(1+t y_{k}\right)} \prod_{m=1}^{N}\left(1+s y_{m}\right)\left(1+t y_{m}\right) \\
& =\frac{s t}{t-s} \sum_{k=1}^{N}\left(\frac{1}{1+s y_{k}}-\frac{1}{1+t y_{k}}\right) \prod_{m=1}^{N}\left(1+s y_{m}\right)\left(1+t y_{m}\right) \\
& =\frac{s t}{t-s}\left(\sum_{m=0}^{N-1}(N-m) e_{m} s^{m} \sum_{n=0}^{N} e_{n} t^{n}-\sum_{m=0}^{N} e_{m} s^{m} \sum_{n=0}^{N-1}(N-n) e_{n} t^{n}\right)
\end{aligned}
$$

(in the expression in Proposition 3.3 for $\delta_{1}$, we replace $t$ by $1 / t$ and multiply by $t^{N-1}$ to get

$$
\left.\sum_{k=1}^{N} \prod_{m \neq k}\left(1+y_{m} t\right)=\sum_{m=0}^{N-1}(N-m) e_{m} t^{m}\right) .
$$

The expression above becomes

$$
\begin{aligned}
\sum_{m, n=0}^{N}(N-m) e_{m} e_{n} s t & \left(\frac{s^{m} t^{n}-s^{n} t^{m}}{t-s}\right) \\
= & \sum_{0 \leqslant m<n \leqslant N}((N-m)-(N-n)) e_{m} e_{n} s t\left(\frac{s^{m} t^{n}-s^{n} t^{m}}{t-s}\right)
\end{aligned}
$$

by skew-symmetry, which in turn equals

$$
\sum_{0 \leqslant m<n \leqslant N} e_{m} e_{n}(n-m) \sum_{k=1}^{n-m} t^{n+1-k} s^{m+k} .
$$

Now we sum over the terms with $n+1-k=i, m+k=j$.

We describe a basis for $P_{n}^{O_{N}}$ which is labelled by the conjugate of the usual labelling (see Macdonald [6, p. 13]). Let $\lambda$ denote an $N$-part (or less) partition, that is, $\lambda=\left(\lambda_{1}, \lambda_{2}, \ldots, \lambda_{N}\right)$ with $\lambda_{i} \in Z_{+}$and $\lambda_{1} \geqslant \lambda_{2} \geqslant \cdots \geqslant \lambda_{N} \geqslant 0$. The weight of $\lambda$ is $|\lambda|:=\sum_{i} \lambda_{i}$.

3.5. Definition. Let $\Omega^{N}$ denote the set of $N$-part partitions, and for each $n=0,1,2, \ldots$ let

$$
\Omega_{n}^{N}:=\left\{\lambda \in \Omega^{N}:|\lambda|=n\right\} \quad \text { and } \quad \partial \Omega_{n}^{N}:=\left\{\lambda \in \Omega_{n}^{N}: \lambda_{1}=\lambda_{2}\right\} .
$$

For $\lambda \in \Omega_{n}^{N}$, define the symmetric function

$$
\phi(\lambda):=e_{1}^{\lambda_{1}-\lambda_{2}} e_{2}^{\lambda_{2}-\lambda_{3}} \cdots e_{N}^{\lambda_{N}} .
$$

For $n=0,1,2, \ldots, \alpha, \beta \geqslant 0$, let

$$
H_{n}^{N}(\alpha, \beta):=P_{2 n}^{O_{N}} \cap \operatorname{ker} L_{\alpha \beta} \quad(n \text { is the } y \text {-degree). }
$$

Note that $\operatorname{deg} \phi(\lambda)=2|\lambda|$ (in the $x$-variables) and $\left\{\phi(\lambda): \lambda \in \Omega_{n}^{N}\right\}$ is a basis for $P_{2 n}^{O_{N}}$. Also $\operatorname{dim} P_{2 n}^{O_{N}}=\left|\Omega_{n}^{N}\right|$, which is the coefficient of $t^{2 n}$ in $\prod_{i=1}^{N}\left(1-t^{2 i}\right)^{-1}$ (the Molien series for $O_{N}$ ).

3.6. Proposition. For each $\alpha, \beta \geqslant 0$,

$$
P_{2 n}^{O_{N}}=\sum_{j=0}^{n} \oplus e_{l}^{j} H_{n-j}^{N}(\alpha, \beta) .
$$


Any continuous $O_{N}$-invariant function on $S$ can be uniformly approximated by polynomials in $\operatorname{ker} L_{\alpha \beta}$.

Proof. Theorem 2.9 applied to this situation gives the first assertion. Now suppose $f$ is continuous and $O_{N}$-invariant on $S$ and $\varepsilon>0$. Then there is a polynomial $p$ such that $|f-p|<\varepsilon$ on $S$. We may assume $p$ is $O_{N}$-invariant (or else we use the projection: sum over all translates of $p$ by elements of $O_{N}$, and divide by $\left.\left|O_{N}\right|\right)$. Now we expand $p$ by degree of homogeneity and use the first part of the proposition (note that $e_{1}=1$ on $S$ ).

We claim that $\operatorname{dim} H_{n}^{N}(\alpha, \beta)=\left|\partial \Omega_{n}^{N}\right|$. Indeed (by Theorem 2.9)

$$
\operatorname{dim} H_{n}^{N}(\alpha, \beta)=\operatorname{dim}\left(P_{2 n}^{O_{N}}\right)-\operatorname{dim}\left(P_{2 n-2}^{O_{N}}\right)=\left|\Omega_{n}^{N}\right|-\left|\Omega_{n-1}^{N}\right|,
$$

and the correspondence $\lambda \rightarrow\left(\lambda_{1}+1, \lambda_{2}, \ldots, \lambda_{N}\right)$ maps $\Omega_{n-1}^{N}$ into $\Omega_{n}^{N}$, with the complement of the image being $\partial \Omega_{n}^{N}$.

This assertion will be explicitly demonstrated by descriptions of elements $\Sigma_{\lambda \in \Omega_{n}^{N}} c(\lambda) \phi(\lambda)$ of $H_{n}^{N}(\alpha, \beta)$ in which the coefficients $c(\lambda), \lambda \in \partial \Omega_{n}^{N}$, can be arbitrarily chosen, determining the other coefficients (this explains our use of the boundary symbol $\partial$ ). To describe $L_{\alpha \beta} \phi(\lambda)$ we introduce operators on $N$-tuples $\lambda$ of integers which change the weight by \pm 1 . For $1 \leqslant i \leqslant j \leqslant N$ and $0 \leqslant k \leqslant(N-j) \wedge$ $(i-1)$, define $U(i, j, k)$ by

$$
(U(i, j, k) \lambda)_{m}=\left\{\begin{array}{l}
\lambda_{m}+1 \text { if } i-k \leqslant m \leqslant i, \\
\lambda_{m}-1 \text { if } j+1 \leqslant m \leqslant j+k, \\
\lambda_{m} \text { otherwise. }
\end{array}\right.
$$

Note that each $U(i, j, k)$ is invertible, $|U(i, j, k) \lambda|=|\lambda|+1$, and $U(i, j, 0)=$ $U(i, i, 0)$ for all $j \geqslant i$. Further, for $\lambda \in \Omega^{N}, U(i, j, k)^{-1} \lambda \in \Omega^{N}$ exactly when $\left(\lambda_{i}-\lambda_{i+1}\right)\left(\lambda_{j}-\lambda_{j+1}\right)>0$ for $k>0$, or $\left(\lambda_{i}-\lambda_{i+1}\right)>0$ for $k=0$.

\subsection{Proposition.}

$$
\begin{aligned}
L \phi(\lambda)= & \sum_{i=2}^{N}\left(\lambda_{i}-\lambda_{i+1}\right)\left(\lambda_{i}-\lambda_{i+1}-1\right) \sum_{k=1}^{(N-i) \wedge(i-1)}(2 k+1) \phi\left(U(i, i, k)^{-1} \lambda\right) \\
& +2 \sum_{\substack{2 \leqslant i<j \leqslant N \\
(N-j) \wedge(i-1)}}\left(\lambda_{i}-\lambda_{i+1}\right)\left(\lambda_{j}-\lambda_{j+1}\right) \\
& \sum_{k=1}^{N}(2 k+j-i+1) \phi\left(U(i, j, k)^{-1} \lambda\right) \\
& +2 \sum_{i=1}^{N}\left(\lambda_{i}-\lambda_{i+1}\right)\left(2 \sum_{j=i}^{N} \lambda_{j}-\left(\lambda_{i}-\lambda_{i+1}\right)-1\right) \phi\left(U(i, i, 0)^{-1} \lambda\right) .
\end{aligned}
$$

Also,

$$
\delta_{1} \phi(\lambda)=\sum_{i=1}^{N}(N-i+1)\left(\lambda_{i}-\lambda_{i+1}\right) \phi\left(U(i, i, 0)^{-1} \lambda\right)
$$


and

$$
\delta_{2} \phi(\lambda)=\sum_{i=1}^{N-1}\left(\begin{array}{c}
N-i+1 \\
2
\end{array}\right)\left(\lambda_{i}-\lambda_{i+1}\right) \phi\left(U(i, i, 0)^{-1} \lambda\right) .
$$

In these formulas, $\lambda_{N+1}=0$.

Proof. This is the result of applying Proposition 3.4 to $\phi(\lambda)$ and a change of index (replace $k$ by $k-j$ ) in the summation there. The third term in $L \phi(\lambda)$ (corresponding to $k=0$ ) is obtained by a telescoping summation over $j \geqslant i$ of the coefficients of $\phi\left(U(i, j, 0)^{-1} \lambda\right)$.

3.8. Proposition. Let $\mu \in \Omega_{n-1}^{N}(n \geqslant 1)$. Then the coefficient of $\phi(\mu)$ in $L_{\alpha \beta} \Sigma_{|\lambda|=n} c(\lambda) \phi(\lambda)$ is

$$
\begin{aligned}
& \sum_{i=1}^{N}\left(\mu_{i}-\mu_{i+1}+1\right)\left(2 \sum_{j=i}^{N} \mu_{j}-\left(\mu_{i}-\mu_{i+1}\right)+(N-i+1)\right. \\
& \left.\cdot\left(\alpha+\frac{1}{2}+(N-i) \beta\right)\right) c(U(i, i, 0) \mu) \\
& +\sum_{i=2}^{N-1}\left(\mu_{i}-\mu_{i+1}+2\right)\left(\mu_{i}-\mu_{i+1}+1\right) \sum_{k=1}^{(N-i) \wedge(i-1)}(2 k+1) c(U(i, i, k) \mu) \\
& +\sum_{2 \leqslant i<j \leqslant N-1}\left(\mu_{i}-\mu_{i+1}+1\right)\left(\mu_{j}-\mu_{j+1}+1\right) \\
& \quad \sum_{k=1}^{(N-j) \wedge(i-1)}(2 k+j-i+1) c(U(i, j, k) \mu)
\end{aligned}
$$

(the sums being extended over those $(i, j, k)$ values for which $U(i, j, k) \mu \in \Omega^{N}$ ).

Proof. We use 3.7 and in each term replace $\lambda$ by the appropriate $U(i, j, k)^{-1} \mu$.

We show how the equations for $c(\lambda)$ resulting from $L_{\alpha \beta} \Sigma_{|\lambda|=n} c(\lambda) \phi(\lambda)=0$ can be solved by an inductive process. The index for this is the degree of $e_{1}$, so we define $r(\lambda):=\lambda_{1}-\lambda_{2}$.

3.9. THEOREM. For any choice of values of $c(\mu)\left(\mu \in \partial \Omega_{n}^{N}\right)$ there is a unique element $\Sigma_{\lambda \in \Omega_{n}^{N}} c(\lambda) \phi(\lambda)$ of $H_{n}^{N}(\alpha, \beta)$, where each $c(\lambda)$ is a linear function in $\left\{c(\mu): \mu \in \partial \Omega_{n}^{N}\right\}$ with the coefficients being rational functions of $\alpha$ and $\beta$ whose denominators are divisors of $r(\lambda) !\left(-2 n+2-N\left(\alpha+\frac{1}{2}+(N-1) \beta\right)\right)_{r(\lambda)}$ (the shifted factorial). The expression for $c(\lambda)(r(\lambda) \geqslant 1)$ is recursively given as an explicit dependence on $c(\mu)$ with $r(\mu)=r(\lambda)-1$ or $r(\lambda)-2$.

Proof. Fix $\lambda \in \Omega_{n}^{N}$ with $r(\lambda)=1$ and let $\mu=U(1,1,0)^{-1} \lambda$ (that is, $\mu_{1}=\lambda_{1}-1$, $\mu_{i}=\lambda_{i}$ for $i \geqslant 2$ ). To get $\Sigma_{|\mu|=n} c(\mu) \phi(\mu) \in H_{n}^{N}$ we set (3.1) equal to zero. The 
coefficient of $c(\lambda)=c(U(1,1,0) \mu)$ is nonzero, indeed it is

$$
r(\lambda)\left(2 n-1-r(\lambda)+N\left(\alpha+\frac{1}{2}+(N-1) \beta\right)\right)
$$

The other $c(\mu)$ which appear in the equation have $r(\mu)<r(\lambda)$, indeed $r(U(i, j, k) \mu)$ $=r(\lambda)-1$ for $2 \leqslant i \leqslant j$ and $k \leqslant(N-j) \wedge(i-1)$ unless $2 \leqslant i \leqslant j \leqslant N-i+2$ and $k=i-2$, in which case $r(U(i, j, i-2))=r(\lambda)-2$.

Admittedly we do not give closed-form expressions for the coefficients $c(\lambda)$, yet they are algorithmically defined. Further, a series $\Sigma_{|\lambda|=n} c(\lambda) \phi(\lambda)$ in $H_{n}^{N}(\alpha, \beta)$ is a generalization of a hypergeometric series (in fact, the ordinary ${ }_{2} F_{1}$-series appears for $N=2$ ), and perhaps there are some summation formulas (which would be values of the functions at the points $(1,1, \ldots, 1),(1, \ldots, 1,0), \ldots,(1,0, \ldots, 0)$ in $y$-coordinates $)$.

Finally, we discuss the weights associated to the groups of type $D_{N}$ generated by the reflections in $\Pi_{i<j}\left(x_{i}^{2}-x_{j}^{2}\right)$, that is, $\alpha=0$ and $\beta \geqslant 1$. It is known that the invariants for $D_{N}$ are generated by $e_{1}, \ldots, e_{N-1}$ (the elementary symmetric functions in $\left\{x_{i}^{2}: 1 \leqslant i \leqslant N\right\}$ as before) and $f_{N}=x_{1} x_{2} \cdots x_{N}$. Note that any invariant polynomial can be uniquely written in the form $p_{0}\left(e_{1}, \ldots, e_{N}\right)+f_{N} p_{1}\left(e_{1}, \ldots, e_{N}\right)$.

3.10. TheOREM. For $h=\left(\Pi_{i<j}\left(x_{i}^{2}-x_{j}^{2}\right)\right)^{\beta}$ and $p$ a polynomial on $\mathbf{R}^{N}, L_{h} p=0$ if and only if $p(x)=p_{0}\left(e_{1}, \ldots, e_{N}\right)+f_{N} p_{1}\left(e_{1}, \ldots, e_{N}\right)$ with $L_{0, \beta} p_{0}=0$ and $L_{1, \beta} p_{1}=0$.

Proof. From Corollary 2.5 we know that for $L_{h} p=0$ it is necessary that $p$ be invariant under the reflection group (reflections in $x_{i}-x_{j}=0, x_{i}+x_{j}=0, i \neq j$ ). Thus $p=p_{0}+f_{N} p_{1}$. By applying the reflection in $x_{1}=0$ to the equation $L_{h} p=0$, we separate the two parts and find that $L_{h} p_{0}=0$ and $L_{h}\left(f_{N} p_{1}\right)=0$. Clearly $L_{h} p_{0}=0$ if and only if $L_{0, \beta} p_{0}=0$. On the other hand, $L_{h}\left(f_{N} p_{1}\right)=L_{h f_{N}} p_{1}+$ $p_{1}\left(L_{h} f_{N}\right)$ (a simple calculation), and

$$
\frac{1}{h} L_{h} f_{N}=\Delta f_{N}+4 \beta \sum_{i<j}\left(\frac{1}{x_{i}^{2}-x_{j}^{2}}\right)\left(x_{i} \frac{\partial}{\partial x_{i}}-x_{j} \frac{\partial}{\partial x_{j}}\right) f_{N}=0 .
$$

Thus $L_{h}\left(f_{N} p_{1}\right)=0$ if and only if $L_{h f_{N}} p_{1}=0$ if and only if $L_{1, \beta} p_{1}=0$.

The group corresponding to $\alpha>0, \beta=0$ is reducible (it is $Z_{2}^{N}$ ); the invariants are arbitrary polynomials in $x_{1}^{2}, \ldots, x_{N}^{2}$ and fall outside the scope of our study.

4. The special cases $N=2$ and $N=3$. In this section we suppress the " $N$ " label on $H_{n}^{N}(\alpha, \beta)$ and $\Omega_{n}^{N}$ since it will be obvious from the context. We discuss the $N=2$ case to show how Jacobi polynomials appear in the theory and as motivation for the search for a differential operator relating adjacent $(\alpha, \beta)$ values (for $\operatorname{ker} L_{\alpha \beta}$ ).

We specialize Proposition 3.8 to $N=2$ and find the coefficient of $\phi\left(\mu_{1}, \mu_{2}\right)$ in $L_{\alpha \beta} \Sigma\left\{c\left(\lambda_{1}, \lambda_{2}\right) \phi\left(\lambda_{1}, \lambda_{2}\right): \lambda_{1}+\lambda_{2}=n, \lambda_{1} \geqslant \lambda_{2} \geqslant 0\right\}$ to be

$$
\begin{array}{r}
\left(\mu_{1}-\mu_{2}+1\right)\left(\mu_{1}+3 \mu_{2}+2 \alpha+2 \beta+1\right) c\left(\mu_{1}+1, \mu_{2}\right) \\
+\left(\mu_{2}+1\right)\left(\mu_{2}+\alpha+\frac{1}{2}\right) c\left(\mu_{1}, \mu_{2}+1\right) .
\end{array}
$$


By Theorem 3.9, $\partial \Omega_{n} \neq \varnothing$ is necessary for $H_{n}(\alpha, \beta) \neq\{0\}$, that is, $n$ must be even, say $n=2 m$, and then $c(m, m)$ can be arbitrary. Set $c(m, m)=1, \mu_{1}=m+j$, $\mu_{2}=m-j-1$ and obtain the recurrence

$$
\begin{aligned}
4(j+1)(2 m+\alpha+\beta-j-1) c(m+j+1, m-j-1) \\
+(m-j)\left(m-j+\alpha-\frac{1}{2}\right) c(m+j, m-j)=0
\end{aligned}
$$

with the solution

$$
c(m+j, m-j)=\frac{(-m)_{j}\left(-m-\alpha+\frac{1}{2}\right)_{j}}{j !(1-2 m-\alpha-\beta)_{j}}\left(\frac{1}{4}\right)^{j} .
$$

Thus $H_{2 m}(\alpha, \beta)$ is spanned by the polynomial

$$
e_{2}^{m} \cdot{ }_{2} F_{1}\left(-m, \frac{1}{2}-m-\alpha ; 1-2 m-\alpha-\beta ; e_{1}^{2} / 4 e_{2}\right),
$$

which in turn (by reversing the order of summation) equals

$$
\left(-\frac{1}{4}\right)^{m} \frac{\left(\alpha+\frac{1}{2}\right)_{m}}{(m+\alpha+\beta)_{m}} e_{1}^{2 m} \cdot{ }_{2} F_{1}\left(-m, m+\alpha+\beta ; \alpha+\frac{1}{2} ; 4 e_{2} / e_{1}^{2}\right) .
$$

The latter is a scalar multiple of $e_{1}^{2 m} P_{m}^{(\alpha-1 / 2, \beta-1 / 2)}\left(1-8 e_{2} / e_{1}^{2}\right)$ (see Szegö [13, p. 62]). The orthogonality structure on the circle is for the weight

$$
\left(x_{1} x_{2}\right)^{2 \alpha}\left(x_{1}^{2}-x_{2}^{2}\right)^{2 \beta} d \omega(x) \text {. }
$$

We express this in $e$-terms as a simple application of these ideas before we treat the harder case $N=3$.

In terms of Jacobians,

$$
d x_{1} d x_{2}=\left(\partial\left(e_{1}, e_{2}\right) / \partial\left(x_{1}, x_{2}\right)\right)^{-1} d e_{1} d e_{2}
$$

in a restricted region of $x$ values, for example $\left\{x_{1}>x_{2}>0\right\}$, which corresponds to $0<e_{2}<e_{1}^{2} / 4$. The unit circle corresponds to $\left\{e_{1}=1\right\}$ so the weight $h^{2} d \omega$ is transformed to (up to a constant multiplier) $e_{2}^{\alpha-1 / 2}\left(1-4 e_{2}\right)^{\beta-1 / 2} d e_{2}$ (note that the discriminant $\left.\left(y_{1}-y_{2}\right)^{2}=e_{1}^{2}-4 e_{2}\right)$, which is of course the appropriate betadistribution for the Jacobi polynomials above.

4.1. A differential relation. The identity

$$
\frac{d}{d t} P_{m}^{(\alpha-1 / 2, \beta-1 / 2)}(t)=\frac{m+\alpha+\beta}{2} P_{m-1}^{(\alpha+1 / 2, \beta+1 / 2)}(t)
$$

(see Szegö [13, p. 63]) suggests that there is a differential operator in the $e$-variables which maps $H_{2 m}(\alpha, \beta)$ to $H_{2 m-2}(\alpha+1, \beta+1)$. This is indeed true and can be interpreted in terms of certain commutators. For $N=2$ we have

$$
L=e_{1} \partial_{1}^{2}+4 e_{2} \partial_{1} \partial_{2}+e_{1} e_{2} \partial_{2}^{2}, \quad \delta_{1}=2 \partial_{1}+e_{1} \partial_{2}, \quad \delta_{2}=\partial_{1}
$$

(see Proposition 3.3). Further, the commutator $\left[\delta_{1}, \delta_{2}\right]=-\partial_{2}$, which commutes with $\delta_{1}$ and $\delta_{2}$, and

$$
\left[L, \partial_{2}\right]=\left[L_{\alpha \beta}, \partial_{2}\right]=-4 \partial_{1} \partial_{2}-e_{1} \partial_{2}^{2}=-\left(\delta_{1}+2 \delta_{2}\right) \partial_{2}
$$


This identity is equivalent to

$$
L_{\alpha+1, \beta+1} \partial_{2}=\left(L_{\alpha \beta}+\delta_{1}+2 \delta_{2}\right) \partial_{2}=\partial_{2} L_{\alpha \beta} .
$$

This shows that $\partial_{2}$ maps $\operatorname{ker} L_{\alpha \beta}$ into $\operatorname{ker} L_{\alpha+1, \beta+1}$ while lowering the degree by 2 . We will look for a similar situation in the case $N=3$.

The case $N=3$. The operators $L, \delta_{1}, \delta_{2}$ are now given by (see Propositions 3.3 and 3.4)

$$
\begin{gathered}
L=e_{1} \partial_{1}^{2}+\left(e_{1} e_{2}+3 e_{3}\right) \partial_{2}^{2}+e_{2} e_{3} \partial_{3}^{2}+4 e_{2} \partial_{1} \partial_{2}+6 e_{3} \partial_{1} \partial_{3}+4 e_{1} e_{3} \partial_{2} \partial_{3} \\
\delta_{1}=3 \partial_{1}+2 e_{1} \partial_{2}+e_{2} \partial_{3} ; \quad \delta_{2}=3 \partial_{1}+e_{1} \partial_{2} .
\end{gathered}
$$

We will use just $\left(\lambda_{2}, \lambda_{3}\right)$ to describe an element $\lambda \in \Omega_{n}$, so $\Omega_{n}$ will be identified with $\left\{\left(\lambda_{2}, \lambda_{3}\right) \in Z^{2}: \lambda_{2} \geqslant \lambda_{3} \geqslant 0,2 \lambda_{2}+\lambda_{3} \leqslant n\right\}$ and $\partial \Omega_{n}=\left\{\left(\lambda_{2}, \lambda_{3}\right) \in \Omega_{n}\right.$ : $\left.\lambda_{3}=n-2 \lambda_{2}\right\}$. We use the function $\mu(n):=\left|\partial \Omega_{n}\right|$ (as in Definition 1.2 of [3]). Observe that $\mu(n)=|\{m \in Z: n / 3 \leqslant m \leqslant n / 2\}|$ and has the values $\mu(1)=0$, $\mu(j)=1$ for $j=0,2,3,4,5$, and $\mu(n+6)=\mu(n)+1$ for $n \geqslant 0$. The latter relation is the most obvious recurrence relation among $\left\{\operatorname{dim} H_{n}(\alpha, \beta)\right\}$. We will exploit this later.

4.2. Proposition. For $n=2,3, \ldots$, elements $\Sigma_{\lambda} c\left(\lambda_{2}, \lambda_{3}\right) \phi\left(\lambda_{2}, \lambda_{3}\right)$ of $H_{n}(\alpha, \beta)$ are obtained by arbitrarily specifying the values $c\left(\lambda_{2}, \lambda_{3}\right)$ for $\lambda_{3}=n-2 \lambda_{2}$ (that is, $\left.\left(\lambda_{2}, \lambda_{3}\right) \in \partial \Omega_{n}\right)$ and then using the recurrence (in the order $\lambda_{1}-\lambda_{2}=n-2 \lambda_{2}-\lambda_{3}$ $=r(\lambda)=1,2, \ldots, n)$

$$
\begin{gathered}
c\left(\lambda_{2}, \lambda_{3}\right)=\left(1 /\left(r(\lambda)\left(r(\lambda)-2 n-\frac{1}{2}-3 \alpha-6 \beta\right)\right)\right) \\
\cdot\left\{\left(\lambda_{2}-\lambda_{3}+1\right)\left(\lambda_{2}+3 \lambda_{3}+2 \alpha+2 \beta+1\right) c\left(\lambda_{2}+1, \lambda_{3}\right)\right. \\
+\left(\lambda_{3}+1\right)\left(\lambda_{3}+\alpha+\frac{1}{2}\right) c\left(\lambda_{2}, \lambda_{3}+1\right) \\
\left.+3\left(\lambda_{2}-\lambda_{3}+2\right)\left(\lambda_{2}-\lambda_{3}+1\right) c\left(\lambda_{2}+1, \lambda_{3}-1\right)\right\},
\end{gathered}
$$

where terms of index outside $\Omega_{n}$ are omitted.

Proof. This is the special case of 3.8 and 3.9 for $N=3$. Note that $c\left(\lambda_{2}, \lambda_{3}\right)$ is a rational function of $\alpha$ and $\beta$ with the denominator being a divisor of

$$
r(\lambda) !\left(\frac{1}{2}-2 n-3 \alpha-6 \beta\right)_{r(\lambda)} .
$$

4.3. Definition. For $\lambda_{2} \geqslant \lambda_{3} \geqslant 0$ let $F_{\lambda_{2}, \lambda_{3}}^{(\alpha, \beta)}$ be the (unique) element of $H_{2 \lambda_{2}+\lambda_{3}}(\alpha, \beta)$ that has $c\left(\lambda_{2}, \lambda_{2}, \lambda_{3}\right)=1$ and $c\left(\lambda^{\prime}\right)=0$ for all $\lambda^{\prime} \in \partial \Omega$ with $\lambda^{\prime} \neq \lambda$ (possible by Proposition 4.2). These functions form a basis for $\operatorname{ker} L_{\alpha \beta}$.

4.4. Special cases for $\alpha, \beta$. From Corollary 2.6 we know that

$$
h(x)=\left(x_{1} x_{2} x_{3}\right)^{\alpha}\left(\prod_{1 \leqslant i<j \leqslant 3}\left(x_{i}^{2}-x_{j}^{2}\right)\right)^{\beta}
$$


is harmonic exactly when $\alpha, \beta$ take the values 0 or 1 ( 4 cases). Thus for any $n \geqslant 0$, each of

$$
\begin{array}{r}
H_{n}(0,0), \quad\left(x_{1} x_{2} x_{3}\right) \cdot H_{n}(1,0), \quad \prod_{i<j}\left(x_{i}^{2}-x_{j}^{2}\right) \cdot H_{n}(0,1), \\
\\
\quad\left(x_{1} x_{2} x_{3} \prod_{i<j}\left(x_{i}^{2}-x_{j}^{2}\right)\right) \cdot H_{n}(1,1)
\end{array}
$$

is a space of ordinary harmonic homogeneous polynomials (of degrees $2 n, 2 n+3$, $2 n+6,2 n+9$, respectively) which are absolutely or relatively (multiplied by \pm 1 ) invariant under the octahedral group $O_{3}$ (they are called "cubic harmonics"). These spaces were studied in [3], but with different bases for the invariants, namely the monomial basis ( $m_{\lambda}$ in Macdonald [6, p. 11]) and a basis related to $S$-functions [6, p. 23]

Because these polynomials are actually harmonic, there are orthogonal projections from the space of all harmonic polynomials onto these subspaces, and these projections can be represented by matrices of Krawtchouk polynomial values.

The weight function in $\left(e_{2}, e_{3}\right)$-space. We require the discriminant

$$
D:=\prod_{i<j}\left(x_{i}^{2}-x_{j}^{2}\right)^{2}
$$

Since $D$ is invariant under $O_{3}$ it must be a polynomial in $e_{1}, e_{2}, e_{3}$, indeed

$$
D=e_{1}^{2} e_{2}^{2}-4 e_{2}^{3}-4 e_{1}^{3} e_{3}-27 e_{3}^{2}+18 e_{1} e_{2} e_{3}
$$

(see van der Waerden [14, p. 102]).

4.5. Proposition. The image of the mapping $\left(x_{1}, x_{2}, x_{3}\right) \rightarrow\left(e_{1}, e_{2}, e_{3}\right)$ of $\mathbf{R}^{3} \rightarrow \mathbf{R}^{3}$ is exactly the set $\left\{\left(e_{1}, e_{2}, e_{3}\right): e_{1}, e_{2}, e_{3}, D \geqslant 0\right\}$.

Proof. The image is obviously contained in this set. Conversely, the cubic polynomial $\left(t+y_{1}\right)\left(t+y_{2}\right)\left(t+y_{3}\right)=t^{3}+e_{1} t^{2}+e_{2} t+e_{3}$ cannot vanish at positive values of $t$ but must have three real roots $-y_{1},-y_{2},-y_{3}$ when $D \geqslant 0$ (see van der Waerden [14, p. 194]), hence each $y_{i} \geqslant 0$ and $x_{i}= \pm \sqrt{y_{i}}$ is real.

We will consider the polynomials in $H_{n}(\alpha, \beta)$ as functions on the domain

$$
E:=\left\{\left(e_{2}, e_{3}\right): e_{2}, e_{3} \geqslant 0, D\left(1, e_{2}, e_{3}\right) \geqslant 0\right\}
$$

(the variable $e_{1}$, while always having the value 1 , will be kept for purposes of homogeneity). For fixed $e_{2}, D$ is a quadratic polynomial in $e_{3}$ allowing the following explicit description of $E$ :

$$
\begin{aligned}
E=\left\{\left(e_{2}, e_{3}\right): 0 \leqslant e_{2} \leqslant \frac{1}{3}, \max \right. & \left(0,9 e_{2}-2-2\left(1-3 e_{2}\right)^{3 / 2}\right) \\
& \left.\leqslant 27 e_{3} \leqslant 9 e_{2}-2+2\left(1-3 e_{2}\right)^{3 / 2}\right\} .
\end{aligned}
$$

From this we find that $E$ is a three-sided figure with the following boundary: a line segment from $(0,0)$ to $\left(\frac{1}{4}, 0\right)$, then a curve (part of $D=0$ ) starting with slope $\frac{1}{2}$ leading to the cusp $\left(\frac{1}{3}, \frac{1}{27}\right)$ (the slope is $\frac{1}{3}$ there), and the third side is the other part of $D=0$, joining $(0,0)$ to $\left(\frac{1}{3}, \frac{1}{27}\right)$ and tangent to the other two sides at these points. 
To change variables in the integrals we prefer to use Cartesian coordinates, rather than spherical polar coordinates, so as not to conceal the important Jacobian $\partial\left(e_{1}, e_{2}, e_{3}\right) / \partial\left(x_{1}, x_{2}, x_{3}\right)$.

4.6. LEMMA. Let $p$ be a polynomial on $\mathbf{R}^{3}$, homogeneous of degree $2 k$. Then

$$
\int_{\mathbf{R}^{3}} p(x) e^{-|x|^{2}} d x=\left(\pi^{3 / 2}\left(\frac{3}{2}\right){ }_{k}\right) \int_{S} p d \omega .
$$

Proof. In polar coordinates $x=r \omega$, where $\omega \in S$ and $r>0$, so we have $d x=$ $4 \pi r^{2} d r d \omega$ (recall $d \omega$ is normalized). Then $p(r \omega)=r^{2 k} p(\omega)$ and the $r$-integral is

$$
\frac{1}{2} \Gamma\left(k+\frac{3}{2}\right)=\frac{1}{2} \Gamma\left(\frac{3}{2}\right)\left(\frac{3}{2}\right)_{k}=\frac{1}{4} \Gamma(1 / 2)\left(\frac{3}{2}\right)_{k} .
$$

4.7. Proposition. Let $f\left(e_{1}, e_{2}, e_{3}\right)$ be a polynomial (considered as a function in $x$-variables). Then

$$
\int_{S} f d \omega=\frac{3}{\pi} \int_{E} f\left(1, e_{2}, e_{3}\right) e_{3}^{-1 / 2} D^{-1 / 2} d e_{2} d e_{3} .
$$

Proof. We may assume $f$ is homogeneous of degree $2 k$ in $x$ (each $e_{i}$ is even). By symmetry,

$$
\begin{aligned}
\int_{\mathbf{R}^{3}} f e^{-|x|^{2}} d x & =48 \int_{x_{1} \geqslant x_{2} \geqslant x_{3} \geqslant 0} f e^{-|x|^{2}} d x \\
& =6 \int_{e_{1}, e_{2}, e_{3}, D \geqslant 0} f\left(e_{1}, e_{2}, e_{3}\right) \exp \left(-e_{1}\right) e_{3}^{-1 / 2} D^{-1 / 2} d e_{1} d e_{2} d e_{3}
\end{aligned}
$$

(because

$$
\frac{\partial\left(e_{1}, e_{2}, e_{3}\right)}{\partial\left(x_{1}, x_{2}, x_{3}\right)}=8 x_{1} x_{2} x_{3} \prod_{i<j}\left(x_{i}^{2}-x_{j}^{2}\right)=8\left(e_{3} D\right)^{1 / 2}
$$

in the specified regions). In the latter integral use the coordinates $\tilde{e}_{2}=e_{2} / e_{1}^{2}$, $\tilde{e}_{3}=e_{3} / e_{1}^{3}$ and the homogeneity relation $f\left(e_{1}, e_{1}^{2} \tilde{e}_{2}, e_{1}^{3} \tilde{e}_{3}\right)=e_{1}^{k} f\left(1, \tilde{e}_{2}, \tilde{e}_{3}\right)$ (as well as one for $D$ ). Then the $e_{1}$-integral is a gamma-function which gives the value

$$
\Gamma\left(k+\frac{3}{2}\right) \int_{E} f\left(1, e_{2}, e_{3}\right)\left(e_{3} D\right)^{-1 / 2} d e_{2} d e_{3} .
$$

Combine this with Lemma 4.6 to get the result.

Selberg (see Askey [1] for the statement and $q$-analogues, Mehta [7, p. 40] for matrix ensemble applications, and Morris [8] for a study of identities related to root systems and such integrals) determined an integral from which one can derive the value of

$$
\int_{\mathbf{R}^{3}}\left(x_{1} x_{2} x_{3}\right)^{2 \alpha}\left(\prod_{i<j}\left(x_{i}^{2}-x_{j}^{2}\right)\right)^{2 \beta} e^{-|x|^{2}} d x
$$


Applying Lemma 4.6 to this result (Askey [1, p. 938]) and using duplication and triplication formulas for $\Gamma$, we obtain

$$
\begin{array}{r}
\int_{S} e_{3}^{\alpha} D^{\beta} d \omega=\frac{(1 / 2)_{\alpha}(1 / 2)_{\alpha+\beta}(1 / 2)_{\alpha+2 \beta}(1 / 2)_{\beta}(1 / 3)_{\beta}(2 / 3)_{\beta}}{(3 / 2)_{3 \alpha+6 \beta}} \cdot 108^{\beta} \\
(\alpha, \beta=0,1,2, \ldots) .
\end{array}
$$

(Thus, we do know the integral of the weight function, but as yet we do not have $L^{2}$-norms of elements of $H_{n}(\alpha, \beta)$.)

Next we describe a third-order differential operator that maps $H_{n}(\alpha, \beta)$ to $H_{n-6}(\alpha+2, \beta+1)$. As some motivation for the lengthy calculation, we point out that the adjoint of $\partial_{2}$ in the case $N=2$ is closely related to the Rodrigues formula for Jacobi polynomials (essentially $P_{n}^{(\alpha, \beta)}$ is the result of applying $\left(\partial_{2}^{*}\right)^{n}$ to $P_{0}^{(\alpha+n, \beta+n)}$; note each factor $\partial_{2}^{*}$ is actually with respect to a different weight function). The structure in $N=3$ is not as simple because $\operatorname{dim} H_{n}(\alpha, \beta)$ increases with $n$ (roughly), nevertheless an analogue of $\partial_{2}^{*}$ will be helpful in giving more structure to $H_{n}(\alpha, \beta)$. To obtain a specific complete orthogonal decomposition remains a subject for further research.

We first sketch the ideas that led to the discovery of the $N=3$ analogue of $\partial_{2}$. By analogy with the $N=2$ case, for each pair $(\alpha, \beta)$ we want a differential operator $T$ (that preserves homogeneous polynomials) and another pair $\left(\alpha^{\prime}, \beta^{\prime}\right)$ such that $\left(L+\left(\alpha^{\prime}+\frac{1}{2}\right) \delta_{1}+2 \beta^{\prime} \delta_{2}\right) T=T\left(L+\left(\alpha+\frac{1}{2}\right) \delta_{1}+2 \beta \delta_{2}\right)$. We write this as

$$
[L, T]+\left(\alpha^{\prime}-\alpha\right) \delta_{1} T+2\left(\beta^{\prime}-\beta\right) \delta_{2} T=-\left(\alpha+\frac{1}{2}\right)\left[\delta_{1}, T\right]-2 \beta\left[\delta_{2}, T\right] .
$$

If $T$ is of order $m$ then the left side is of order $\leqslant m+1$, whereas the right side is of order $m$. Thus a necessary condition for such an operator is that the $(m+1)$ st-order terms of $[L, T]+c_{1} \delta_{1} T+c_{2} \delta_{2} T$ vanish for some choice of $c_{1}, c_{2}$. With this, one can rule out first-order operators fairly quickly.

Another important consideration is the dimension pattern mentioned above, namely, $\operatorname{dim} H_{n}(\alpha, \beta)=\operatorname{dim} H_{n-6}\left(\alpha^{\prime}, \beta^{\prime}\right)+1$, so an operator that lowers the degree by 6 should have good properties. Finally, one has the feeling that $\partial_{1}$ should not appear (is $e_{1}$ not constant on $E$ ?). Thus one arrives at trying a linear combination of $\partial_{2}^{3}, e_{1} \partial_{2}^{2} \partial_{3}, e_{2} \partial_{2} \partial_{3}^{2}, e_{3} \partial_{3}^{3}, \partial_{3}^{2}$.

We let

$$
T=c_{1} \partial_{2}^{3}+c_{2} e_{1} \partial_{2}^{2} \partial_{3}+c_{3} e_{2} \partial_{2} \partial_{3}^{2}+c_{4} e_{3} \partial_{3}^{3},
$$

and after lengthy calculations find that the fourth-order terms in

$$
[L, T]+\left(c_{5} \partial_{1}+c_{6} e_{1} \partial_{2}+c_{7} \partial_{3}\right) T
$$

are cancelled when $c_{1}=c_{2}=c_{3}=c_{4}=1, c_{5}=12, c_{6}=6, c_{7}=2$ (there are 12 fourth-order terms giving 12 fortunately consistent equations for $c_{1}$ through $c_{7}$ ). Also $12 \partial_{1}+6 e_{1} \partial_{2}+2 e_{2} \partial_{3}=2\left(\delta_{1}+\delta_{2}\right)$.

4.8. Definition. Let $T:=\partial_{2}^{3}+e_{1} \partial_{2}^{2} \partial_{3}+e_{2} \partial_{2} \partial_{3}^{2}+e_{3} \partial_{3}^{3}$. This is a third-order operator that lowers the degree of a polynomial by 6 . 
4.9. Proposition. $\left.[L, T]+2 \dot{(} \delta_{1}+\delta_{2}\right) T=-2\left[L, \partial_{3}^{2}\right]-4\left(\delta_{1}+\delta_{2}\right) \partial_{3}^{2}=4\left[\delta_{2}, T\right]$, and $\left[\delta_{1}, T\right]=0$.

Proof. We sketched the argument showing that the fourth-order terms on the left side cancel out. Calculating the remaining (all third-order) terms we obtain $4\left(3 \partial_{2}+e_{1} \partial_{3}\right) \partial_{2} \partial_{3}$. Further,

$$
\begin{aligned}
{\left[L, \partial_{3}^{2}\right] } & =-6 \partial_{2}^{2} \partial_{3}-\left(12 \partial_{1}+8 e_{1} \partial_{2}+2 e_{2} \partial_{3}\right) \partial_{3}^{2} \\
& =-2\left(3 \partial_{2}+e_{1} \partial_{3}\right) \partial_{2} \partial_{3}-2\left(\delta_{1}+\delta_{2}\right) \partial_{3}^{2},
\end{aligned}
$$

$\left[\delta_{2}, T\right]=\left(3 \partial_{2}+e_{1} \partial_{3}\right) \partial_{2} \partial_{3}$, and $\left[\delta_{1}, T\right]=0$ are all straightforwardly obtained.

4.10. Definition. Let $T_{\beta}:=T+(\beta+2) \partial_{3}^{2}$.

4.11. TheOREM. For each $n \geqslant 6, T_{\beta}$ maps $H_{n}(\alpha, \beta)$ onto $H_{n-6}(\alpha+2, \beta+1)$. The kernel of $T_{\beta}$ is spanned by

$$
\sum_{j} \frac{(-1)^{j}(p-m)_{3 j}}{(p+2 j) !(1-m-\beta)_{j}} F_{m-j . p+2 j}^{(\alpha, \beta)},
$$

where $n=2 m+p($ with $m=[n / 2], p=0$ or 1$)$ and $0 \leqslant j \leqslant(m-p) / 3$.

Proof. We find $c$ so that

$$
\left[L, T+c \partial_{3}^{2}\right]+2\left(\delta_{1}+\delta_{2}\right)\left(T+c \partial_{3}^{2}\right)=-\left(\alpha+\frac{1}{2}\right)\left[\delta_{1}, T+c \partial_{3}^{2}\right]-2 \beta\left[\delta_{2}, T+c \partial_{3}^{2}\right]
$$

is an identity (see (4.1)). By the relations above, this becomes $(4-2 c)\left[\delta_{2}, T\right]=$ $-2 \beta\left[\delta_{2}, T\right]$, so that $c=\beta+2$ is the solution (note $\left[\delta_{1}, \partial_{3}^{2}\right]=0=\left[\delta_{2}, \partial_{3}^{2}\right]$ ). Thus $L_{\alpha+2, \beta+1} T_{\beta}=T_{\beta} L_{\alpha, \beta}$, and $T_{\beta}$ lowers the degree of a polynomial by 6 .

The elements of $\partial \Omega_{n}$ are given by $\{(m-j, p+2 j): m=[n / 2], p=n-2 m$, $0 \leqslant j \leqslant(m-p) / 3\}$, so suppose $T_{\beta} \Sigma_{j} c_{j} F_{m-j, p+2 j}^{(\alpha, \beta)}=0$. Since the left side is in $H_{n-6}(\alpha+2, \beta+1)$, it suffices to require (by Proposition 4.2) that the coefficients of $e_{2}^{\lambda_{2}-\lambda_{3}} e_{3}^{\lambda_{3}}\left(2 \lambda_{2}+\lambda_{3}=n-6\right)$ be zero. Such terms can only come from $e_{2}^{\mu_{2}-\mu_{3}} e_{3}^{\mu_{3}}$ $\left(2 \mu_{2}+\mu_{3}=n\right)$ (since there is no $\partial_{1}$ in $\left.T_{\beta}\right)$.

Indeed

$$
\begin{aligned}
T_{\beta} e_{2}^{\mu_{2}-\mu_{3}} e_{3}^{\mu_{3}}= & \left(\mu_{2}-\mu_{3}\right)\left(\mu_{2}-\mu_{3}-1\right)\left(\mu_{2}-\mu_{3}-2\right) \cdot e_{2}^{\mu_{2}-\mu_{3}-3} e_{3}^{\mu_{3}} \\
& +\mu_{3}\left(\mu_{3}-1\right)\left(\mu_{2}+\beta\right) e_{2}^{\mu_{2}-\mu_{3}} e_{3}^{\mu_{3}-2}\left(+ \text { a term involving } e_{1}\right) .
\end{aligned}
$$

Put $\mu_{2}=m-j, \mu_{3}=p+2 j$ in this relation and get another formula by replacing $j$ by $j+1$. The equation $T_{\beta} \sum c_{j} F_{m-j, p+2 j}^{(\alpha, \beta)}=0$ now gives a simple recurrence for $c_{j}$ with a unique solution (with $c_{0}=1$ ) (the recurrence for $c_{j+1}$ in terms of $c_{j}$ is the coefficient of $\left.e_{2}^{m-p-3 j-3} e_{3}^{p+2 j}\right)$. Since $\left(\operatorname{ker} T_{\beta}\right) \cap H_{n}(\alpha, \beta)$ is of dimension $1, T_{\beta}$ must be onto $H_{n-6}(\alpha+2, \beta+1)$.

Note the difference for $N=3$ and $N=2$ in the contiguous $(\alpha, \beta)$ values. Observe, however, that in $N=2$ the weight function $e_{2}^{\alpha}\left(e_{1}^{2}-4 e_{2}\right)^{\beta}$ goes up by degree 2 in each factor, and in $N=3$ the weight $e_{3}^{\alpha} D^{\beta}$ in going to $e_{3}^{\alpha+2} D^{\beta+1}$ has each factor's degree increased by 6 .

From the general theory (Proposition 3.6) we know $H_{n}(\alpha, \beta) \perp H_{m}(\alpha, \beta)$ for $m \neq n$ in the Hilbert space $L^{2}(E ; \alpha, \beta):=L^{2}\left(E, e_{3}^{\alpha-1 / 2} D^{\beta-1 / 2} d e_{2} d e_{3}\right)$, and $L^{2}(E ; \alpha, \beta)=\sum_{n=0}^{\infty} \oplus H_{n}(\alpha, \beta)$ (complete direct sum). Thus the operator $T_{\beta}$ is 
densely defined on $L^{2}(E ; \alpha, \beta)$ with dense range in $L^{2}(E ; \alpha+2, \beta+1)$, so it is of great interest to determine $T_{\beta}^{*}$ (Rasala [10] did a detailed analysis of the role of adjoints in one-variable polynomials).

For $f, g$ polynomials in $e_{2}, e_{3}$ we have the formal identity

$$
\begin{aligned}
\int_{E} f & \left(T_{\beta}^{*} g\right) e_{3}^{\alpha-1 / 2} D^{\beta-1 / 2} d e_{2} d e_{3}=\int_{E}\left(T_{\beta} f\right) g e_{3}^{\alpha+3 / 2} D^{\beta+1 / 2} d e_{2} d e_{3} \\
& =-\int_{E} f\left\{\left(\partial_{2}^{3}+e_{3} \partial_{3}^{3}+e_{2} \partial_{2} \partial_{3}^{2}+e_{1} \partial_{2}^{2} \partial_{3}+(2-\beta)\right) \cdot\left(g e_{3}^{\alpha+3 / 2} D^{\beta+1 / 2}\right)\right\} d e_{2} d e_{3} ;
\end{aligned}
$$

the latter equation comes from three-fold integration by parts provided that $\left(g e_{3}^{\alpha+3 / 2} D^{\beta+1 / 2}\right)$ vanishes of sufficiently high order on the boundary of $E$, so temporarily we assume $\alpha \geqslant 1, \beta \geqslant 3$. We define formally

$$
T_{\beta}^{*} g:=-\left(T_{(-\beta)}\left(g e_{3}^{\alpha+3 / 2} D^{\beta+1 / 2}\right)\right) e_{3}^{1 / 2-\alpha} D^{1 / 2-\beta} .
$$

This is indeed the adjoint of $T_{\beta}$ for smooth $g$ and possibly restricted $\alpha, \beta$. However, it is not at all obvious that $T_{\beta}^{*}$ maps polynomials to polynomials, although the degree of (positive) homogeneity is raised by 6 . The heavy part of the following calculation is dealing with derivatives of $D^{\beta+1 / 2}$. Grouping by $\alpha$-terms, we have

$$
\begin{aligned}
T_{\beta}^{*} g= & -e_{3}^{2}\left(P_{0} g+P_{3} \partial_{3} g+2 P_{6} \partial_{3}^{2} g+e_{3} D \partial_{3}^{3} g\right. \\
& \left.+P_{2} \partial_{2} g+2 P_{5} \partial_{2} \partial_{3} g+e_{2} D \partial_{2} \partial_{3}^{2} g+e_{1} D \partial_{2}^{2} \partial_{3} g+D \partial_{2}^{3} g\right) \\
& -\left(-\alpha-\frac{3}{2}\right) e_{3}\left(P_{3} g+2 P_{6} \partial_{3} g+3 e_{3} D \partial_{3}^{2} g+2 P_{5} \partial_{2} g+2 e_{2} D \partial_{2} \partial_{3} g\right) \\
& -\left(-\alpha-\frac{3}{2}\right)_{2}\left(P_{6} g+3 e_{3} D \partial_{3} g+e_{2} D \partial_{2} g\right)-\left(-\alpha-\frac{3}{2}\right)_{3} D g
\end{aligned}
$$

where (subscripts indicate degree)

$$
\begin{aligned}
& P_{0}:=\left(\left(\partial_{2}^{3}+e_{1} \partial_{2}^{2} \partial_{3}+e_{2} \partial_{2} \partial_{3}^{2}+e_{3} \partial_{3}^{3}+(2-\beta) \partial_{3}^{2}\right) D^{\beta+1 / 2}\right) D^{1 / 2-\beta}, \\
& P_{3}:=\left(\left(e_{1} \partial_{2}^{2}+2 e_{2} \partial_{2} \partial_{3}+3 e_{3} \partial_{3}^{2}+2(2-\beta) \partial_{3}\right) D^{\beta+1 / 2}\right) D^{1 / 2-\beta}, \\
& P_{6}:=\left(\left(e_{2} \partial_{2}+3 e_{3} \partial_{3}+(2-\beta)\right) D^{\beta+1 / 2}\right) D^{1 / 2-\beta}, \\
& P_{2}:=\left(\left(3 \partial_{2}^{2}+2 e_{1} \partial_{2} \partial_{3}+e_{2} \partial_{3}^{2}\right) D^{\beta+1 / 2}\right) D^{1 / 2-\beta}, \\
& P_{5}:=\left(\left(e_{1} \partial_{2}+e_{2} \partial_{3}\right) D^{\beta+1 / 2}\right) D^{1 / 2-\beta} .
\end{aligned}
$$

It remains to calculate the functions $P_{j}$. They are in fact polynomials. The differentiations result in polynomials (in $e_{1}, e_{2}, e_{3}$ ) which have to be divided by $D$. (The author carried out this polynomial arithmetic on his APPLE II computer.) The results are:

$$
\begin{aligned}
& P_{0}=-6(2 \beta+1)(3 \beta+1)(3 \beta+2), \\
& P_{3}=2(2 \beta+1)\left\{6(\beta+1)\left(e_{1} e_{2}-9 e_{3}\right)-(\beta+2)\left(e_{1}^{2}-3 e_{2}\right) e_{1}\right\}, \\
& P_{6}=5(\beta+1) D+2(2 \beta+1)\left(e_{1}^{2}-3 e_{2}\right)\left(3 e_{1} e_{3}-e_{2}^{2}\right), \\
& P_{2}=6(2 \beta+1)(3 \beta+2)\left(e_{1}^{2}-3 e_{2}\right), \\
& P_{5}=(2 \beta+1)\left(9 e_{3}-e_{1} e_{2}\right)\left(e_{1}^{2}-3 e_{2}\right) .
\end{aligned}
$$


Note that the inner bracketed expressions in $e_{1}, e_{2}, e_{3}$ all vanish at $e_{1}=1, e_{2}=\frac{1}{3}$, $e_{3}=\frac{1}{27}$ (the cusp).

4.12. ThEOREM. The adjoint of $T_{\beta}$ on polynomials in $L^{2}(E ; \alpha+2, \beta+1)$ is given by (4.3) and maps $H_{n}(\alpha+2, \beta+1)$ into $H_{n+6}(\alpha, \beta)$. This holds for all $\alpha, \beta \geqslant 0$.

Proof. Let $g \in H_{n}(\alpha+2, \beta+1)$ and let $f$ be a polynomial of degree $<n+6$. By definition,

$$
\int_{E}\left(T_{\beta}^{*} g\right) f e_{3}^{\alpha-1 / 2} D^{\beta-1 / 2} d e_{2} d e_{3}=\int_{E} g\left(T_{\beta} f\right) e_{3}^{\alpha+3 / 2} D^{\beta+1 / 2} d e_{2} d e_{3} .
$$

But $T_{\beta} f$ is a polynomial of degree $<n$, so, by the orthogonal decomposition of $L^{2}(E ; \alpha+2, \beta+1)$, the latter integral is zero. Thus $T_{\beta}^{*} g$ is a homogeneous polynomial of degree $n+6$ which is orthogonal to all lower-degree polynomials in $L^{2}(E ; \alpha, \beta)$, hence $T_{\beta}^{*} g \in H_{n+6}(\alpha, \beta)$. The identity (4.2) holds for any two polynomials $f, g$ for infinitely many values of $\alpha, \beta$ so it will hold for all $\alpha, \beta$ for which both sides are finite (this includes $\alpha, \beta \geqslant 0$ ).

4.13. Corollary. For $n \geqslant 6, T_{\beta}^{*} H_{n-6}(\alpha+2, \beta+1)$ is contained in the subspace of $H_{n}(\alpha, \beta)$ of functions that vanish at $(0,0) \in E$; that is, they have no $e_{1}^{n}$ term.

Proof. A detailed examination of $T_{\beta}^{*} g$ in (4.3) shows that every term contains one of the following as a multiplier: $e_{3}, D, 3 e_{1} e_{3}-e_{2}^{2}$ (and $D$ itself has no $e_{1}^{6}$ term). Each of these vanishes at $(0,0)$.

Of course we now have an orthogonal decomposition of $H_{n}(\alpha, \beta)$ as $\operatorname{ker} T_{\beta} \oplus$ $T_{\beta}^{*} H_{n-6}(\alpha+2, \beta+1)$. One would like an explicit orthogonal decomposition of $H_{n}(\alpha, \beta)$ into one-dimensional subspaces. Possibly one can explicitly find the eigenvectors of $T_{\beta}^{*} T_{\beta}$. The computations for this at least can be restricted to considering the coefficients of $\phi(\lambda)$ with $\lambda_{1}=\lambda_{2}$. One also desires $L^{2}$-norms and special values $\left(\right.$ at $\left.(0,0),\left(\frac{1}{4}, 0\right),\left(\frac{1}{3}, \frac{1}{27}\right)\right)$ of such eigenvectors. Besides these basic problems there are, as usual, the questions about relations among polynomials of different $(\alpha, \beta)$ families and known families of one- or two-variable polynomials.

It does seem that the polynomials of this paper are a quite natural analogue of the Jacobi polynomials, unifying the fundamental concepts of reflection groups, harmonic functions, and multivariate beta distributions.

\section{REFERENCES}

1. R. Askey, Some basic hypergeometric extensions of integrals of Selberg and Andrews, S.I.A.M J. Math. Anal. 11 (1980), 938-951.

2. H. S. M. Coxeter, Regular polytopes, 3rd ed., Dover, New York, 1973.

3. C. F. Dunkl, Cube group invariant spherical harmonics and Krawtchouk polynomials, Math. Z. 177 (1981), 561-577.

4. T. Koornwinder, Two-variable analogues of the classical orthogonal polynomials, Theory and Applications of Special Functions (R. Askey, ed.), Academic Press, New York, 1975, pp. 435-495.

5. O. Laporte, Polyhedral harmonics, Z. Naturforsch. 3a (1948), 447-456.

6. I. G. Macdonald, Symmetric functions and Hall polynomials, Clarendon Press, Oxford, 1979.

7. M. L. Mehta, Random matrices and the statistical theory of energy levels, Academic Press, New York, 1967.

8. W. G. Morris II, Constant term identities for finite and affine root systems: Conjectures and theorems, Ph. D. Thesis, Univ. of Wisconsin, Madison, 1982. 
9. G. Prasad, Über das Gauss'sche Verfahren für die Zerlegung einer ganzen homogenen Funktion in Kugelfunktionen, Math. Ann. 72 (1912), 435-436.

10. R. Rasala, The Rodrigues formulas and polynomial differential operators, J. Math. Anal. Appl. 84 (1981), 443-482.

11. R. Stanley, Invariants of finite groups and their applications to combinatorics, Bull. Amer. Math. Soc. (N.S.) 1 (1979), 475-511.

12. R. Steinberg, Invariants of finite reflection groups, Canad. J. Math. 12 (1960), 616-618.

13. G. Szegö, Orthogonal polynomials, Amer. Math. Soc. Colloq. Publ., vol. 23, Amer. Math. Soc., Providence, R. I., 1959.

14. B. L. van der Waerden, Algebra. Vol. 1, 7th ed., Springer-Verlag, Berlin and New York, 1966.

Department of Mathematics, University of Virginia, Charlottesville, Virginia 22903 